\section{PSICOLOGIA IBEROAMERICANA}

\section{Psicología lberoamericana}

ISSN: 1405-0943

psicología.iberoamericana@uia.mx

Universidad Iberoamericana, Ciudad de

México

México

Delgadillo-Hernández, Alma Olga; López-Coutiño, Berenice; Cabral-Gallo, María del Consuelo;

Sánchez-Zubieta, Fernando Antonio

Funcionamiento familiar de adolescentes con leucemia

Psicología Iberoamericana, vol. 20, núm. 1, enero-junio, 2012, pp. 48-62

Universidad Iberoamericana, Ciudad de México

Distrito Federal, México

Disponible en: http://www.redalyc.org/articulo.oa?id=133924623006

Cómo citar el artículo

- Número completo

- Más información del artículo

- Página de la revista en redalyc.org

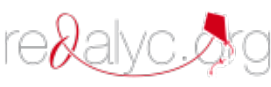

Sistema de Información Científica

Red de Revistas Científicas de América Latina, el Caribe, España y Portugal

Proyecto académico sin fines de lucro, desarrollado bajo la iniciativa de acceso abierto 


\title{
Funcionamiento familiar de adolescentes con leucemia
}

\author{
Family Functioning of Adolescents with Leukemia
}

\author{
Alma Olga Delgadillo-Hernández* \\ Instituto de InVESTIGACIÓN DE CÁNCER EN LA INFANCIA Y AdolesCENCIA, \\ Centro Universitario de Ciencias de la Salud, Universidad de Guadalajara, México \\ Berenice López-Coutiño \\ Unidad de Auditoría Permanente Académica, Universidad Guadalajara lamar, México \\ María del Consuelo Cabral-Gallo \\ Fernando Antonio Sánchez-Zubieta \\ Instituto de InVESTIGACIÓN de Cáncer en la Infancia y Adolescencia, \\ Centro Universitario de Ciencias de la Salud, Universidad de Guadalajara, México
}

\section{RESUMEN}

El objetivo de este estudio fue describir el funcionamiento familiar en adolescentes que padecen leucemia. Se empleó metodología cualitativa. Fueron 21 participantes en total, tres mujeres y un hombre diagnosticados con algún tipo de leucemia; sus edades oscilaban entre los 13 y 16 años, y los otros 18 fueron los padres y otros miembros de las familias de los adolescentes. La recolección de los datos fue por medio de entrevistas semiestructuradas que fueron audiograbadas. El análisis de los datos fue temático. Como parte de los resultados se encontraron alteraciones en el funcionamiento familiar que se manifiestaban en la jerarquía, roles y límites difusos al inicio del diagnóstico, así como un desequilibrio del sistema que oscila entre uno y dos meses aproximadamente; otro hallazgo fue que la búsqueda de atención y el apoyo por parte de la familia posibilitaba la presencia de adaptación y reorganización del sistema familiar, logrando jerarquías, roles y límites claros. Podemos concluir que en el inicio de la enfermedad las familias presentan alteraciones en su funcionamiento, no obstante, aparece una fase de adaptación y de reorganización del sistema.

Descriptores: funcionamiento familiar, leucemia, familia, cáncer, adolescentes.

\section{ABSTRACT}

The aim of this study was to describe family functioning in adolescents with leukemia. Qualitative methodology was used. Were 21 participants in total, three women and one man diagnosed with some kind of leukemia, their ages ranged between 13 and 16, the rest of the participants were parents and other family members of adolescents with leukemia. The data collection was through semi-structured interviews and there were audio recorded. Data analysis was thematic. Changes in family functioning that manifest themselves in the hierarchy. Parts of the results presented were roles and boundaries which are blurred at the beginning of diagnosis. Also, there is an imbalance of the system ranges from one to two months; we found that the search care and support from family presence allows for adaptation and reorganization of the family system, making hierarchies, roles and boundaries clear. We concluded that the onset of the disease, families present alterations in their function; however, there is a phase of adaptation and reorganization of the system.

Keywords: family functioning, leukemia, family, cancer, teenagers.

\footnotetext{
* Para correspondencia: Alma Olga Delgadillo Hernández. Instituto de Investigación de Cáncer en la Infancia y Adolescencia. Salvador Quevedo y Zubieta núm. 750, Guadalajara, Jal., tel: 333-105-1103, e-mail: alma67@yahoo.com. Berenice López Coutiño. Unidad de Auditoría Permanente Académica. Av. Vallarta núm. 3273, Guadalajara, Jal., tel. 331-067-9483, e-mail: psique43@hotmail.com. María del Consuelo Cabral Gallo. Instituto de Investigación de Cáncer en la Infancia y Adolescencia. Salvador Quevedo y Zubieta núm. 750, Guadalajara, Jal., tel: 333-116-5641, e-mail: consuelo1205@hotmail.com. Fernando Antonio Sánchez Zubieta. Instituto de Investigación de Cáncer en la Infancia y Adolescencia. Salvador Quevedo y Zubieta núm. 750, Guadalajara, Jal., tel. 331-466-3092, e-mail: drfernandos59@hotmail.com.
} 


\section{INTRODUCCIÓN}

Enfrentar la enfermedad grave de un familiar, que puede ser mortal, y el sufrimiento que ésta conlleva es una tarea difícil, y cuando el enfermo es un adolescente intervienen otros factores que hacen más complicado este proceso. Con el avance de la enfermedad, la familia va experimentando una serie de sentimientos potencialmente estresantes: culpa, rabia, hostilidad, desprotección e inutilidad, dolor, tristeza, desasosiego, temor, desesperanza, confusión y depresión son los más frecuentes (Die-Trill, 1987); el sistema familiar tendrá que hacer ajustes emocionales, económicos y prácticos, reestructurar sus funciones, modificar las relaciones entre sus miembros, sus valores y sus expectativas a futuro (Velasco \& Sinibaldi, 2001).

Los adolescentes adquieren normas, costumbres y formas de interrelación en los ámbitos familiar, escolar y comunitario, pero la familia es uno de los entornos más significativos (Fernández \& Musito, 2007), por ello, es necesario investigar el impacto en el funcionamiento de ésta cuando uno de sus miembros se encuentra transitando de la niñez a la edad adulta y es diagnosticado con algún tipo de cáncer.

Según Cuevas, Villasis y Fajardo (2003), en las últimas dos décadas el incremento de las enfermedades crónicas ha sido alarmante. Por una parte, hay una mayor exposición a riesgos debido a los estilos de vida poco saludables en las familias; por la otra, las poblaciones adolescentes están más expuestas al sufrimiento de alguna enfermedad, como el cáncer, que se manifiesta en como leucemia (Fajardo, Mejía, Hernández, Mendoza, Garduño \& Martínez, 1999). El interés de este estudio en las familias con adolescentes que padecen leucemia se debe a su curso prolongado, progresivo, con deterioro físico, mental o ambos, que altera el funcionamiento regular de la familia, y que en muchos de los casos tiene como desenlace la muerte.

La presencia de enfermedades como el cáncer repercute en el sistema familiar (Barreto, Molero \& Pérez, 2000; Meléndez, 2005; Ramírez, 2008), además de tener que afrontar grandes niveles de estrés, de las diversas creencias que puedan tener acerca de la enfermedad y de la muerte otros miembros de la familia, así como de la presencia de diversos mecanismos defensivos como la negación o la evasión, que favorecen la adopción de actitudes desfavorables para la adherencia al tratamiento, recuperación y mejora del miembro enfermo (Die-Trill, 1987; Navarro, 2004; Méndez, 2007; Pérez \& Rodríguez, 2005).

Pese a la gravedad y las consecuencias que ocasiona la leucemia, se ha estudiado el cáncer en la infancia y la adolescencia, tomando en cuenta la participación del individuo y de su familia desde diferentes enfoques; entre los más significativos se encuentran la terapia familiar sistémica, el modelo biopsicosocial y el modelo de enfermedad crónica (Kornblit, 1984; Minuchin, 1985). El primero abona al estudio del curso de la enfermedad, el cual está condicionado por sus características propias y por el modo en que la familia y el paciente la afrontan (Gasulla, 2009).

Para este trabajo se eligió el Modelo Estructural de Minuchin para el estudio del funcionamiento familiar; dicho modelo centra su atención en el concepto normativo de familia sana, en el cual se propone un desarrollo ideal para el funcionamiento adecuado de la familia. Reconoce diferentes subsistemas (individual, conyugal, parental, fraterno) con funciones específicas, así como el mantenimiento de una jerarquía clara basada en la competencia parental para decidir los asuntos relacionados con la familia, límites claramente dibujados entre los subsistemas y fronteras flexibles (Minuchin, 1985; Umbarger, 1983).

Un funcionamiento familiar saludable es aquel que le posibilita a la familia cumplir con éxito con los objetivos y funciones que le están histórica y socialmente asignados (Atri, 2006; Caqueo \& Lemos, 2008; Ccoicca, 2010; Herrera, 1997; Minuchin \& Fishman, 1984), tales como: satisfacción de las necesidades afectivo emocionales y materiales de sus miembros, transmisión de valores éticos y culturales, promoción y facilitación del proceso de socialización de sus miembros, establecimiento y mantenimiento de un equilibrio que sirva para enfrentar las tensiones que se producen en el curso del ciclo vital, integración de patrones para las relaciones interpersonales, la creación de las condiciones propicias para el desarrollo de la identidad personal y la adquisición de la identidad sexual (Herrera, 1997; Navarro, 2004; Ortiz \& Louro, 1996; Ortiz, 1999).

Entre el 2003 y 2004, en Cuba, Pérez y Rodríguez (2005) estudiaron a 25 familias que tuvieran un miembro con diagnóstico de enfermedad oncológica, con el objetivo de determinar la repercusión de éste en la 
familia. Concluyeron que la mayoría de las familias otorgan a la enfermedad oncológica algún nivel de repercusión, generalmente en sentido positivo, ya que en casi todas se presentó un evento simultáneo al proceso del cáncer; por ejemplo, nuevos nacimientos y salida de un miembro de la familia en la búsqueda de una mejora económica, que en otras circunstancias o momentos de la vida familiar serían evaluadas de diferente manera.

Aguilar (2007) realizó un estudio con un grupo de madres de pacientes de entre 0 y 18 años con diagnóstico de neoplasias malignas. El objetivo fue establecer la relación entre el funcionamiento familiar y las siguientes variables: tiempo de diagnóstico de la enfermedad, edad del niño o adolescente, escolaridad, número de hijos y relación de pareja de la madre, así como determinar la percepción que tienen las madres del apoyo social e identificar sus estrategias de afrontamiento; infirieron que no se encontró dependencia entre el funcionamiento familiar y las variables con que fue relacionado.

Muñoz y Paredes (2006) realizaron un estudio en Chile con siete familias que tenían un hijo con diagnóstico de cáncer; el objetivo fue conocer la percepción de los padres con respecto al funcionamiento familiar en los primeros meses después de un diagnóstico de leucemia. Los resultados sugieren la necesidad de establecer un nuevo orden del funcionamiento familiar a partir de que el diagnóstico de la enfermedad genera modificaciones, que se reflejan sobre todo en la figura de los padres, quienes se ven susceptibles y vulnerables ante la aparición del padecimiento y deben generar cambios en función de las reglas, jerarquías, límites y roles, con el fin de cubrir las necesidades del hijo enfermo y mantener el equilibrio familiar.

Velasco y Sinibaldi (2001) incursionan en el estudio del impacto del diagnóstico de una enfermedad crónica en la familia; explicaron los efectos del padecimiento sobre el grupo familiar desde un punto de vista sistémico. El modelo de enfermedad crónica desarrollado por Rolland describe los aspectos psicosociales propios de una enfermedad crónica a través de tres fases: crisis inicial, crónica y terminal; éstas enmarcan el funcionamiento familiar, señalan la presencia de ajuste y adaptación, dependencia y sobreprotección, además de la integración de la red social de apoyo de la familia (Navarro, 2004).
Al respecto, los estudios realizados en México por Ramírez (2008) y López, Sauceda y Cuevas (2006) parecen consistentes e identifican un funcionamiento disfuncional en familias que presentan una enfermedad crónica cómo el cáncer, desarrollándose desde enfoques epidemiológicos que no rescatan la subjetividad, la experiencia de los(as) pacientes y la perspectiva bajo la cual interactúan con su familia.

Otros estudios han identificado alteraciones en el funcionamiento familiar debido al cáncer en los roles familiares y el estilo de vida, presiones financieras, preocupación sobre cómo cuidar al paciente, inadecuada percepción de los servicios de apoyo y conflictos entre los distintos miembros de la familia (Barreto et al., 2000; Muñoz \& Paredes, 2006; Velasco Sinibaldi, 2001). Por su parte, Meléndez (2005), señala la presencia de un funcionamiento disfuncional en forma de rigidez, poca adaptación a la enfermedad, límites y jerarquías difusas, así como roles débilmente claros. No obstante, estos estudios provenientes de una metodología cualitativa no reflejan la perspectiva de las familias mexicanas, ni las particularidades de su cultura.

\section{MÉTODO}

Se realizó un estudio cualitativo descriptivo con familias de adolescentes con diagnóstico de leucemia que acudieron al opd Hospital Civil de Guadalajara "Dr. Juan I. Menchaca" (HCGJIM). El lugar de estudio fue el Servicio de Hemato-Oncología Pediátrica.

Para la selección de los informantes, se contactó a las familias de adolescentes con diagnóstico de leucemia en las instalaciones del hospital. Los criterios de selección fueron: a) diagnóstico de leucemia de no más de tres meses de antigüedad, $b$ ) ser usuario del HCGJIM, c) la inclusión de los padres, hermanos adolescentes y cualquier miembro consanguíneo (adolescente o adulto) que cohabitara con los mismos, $d$ ) rango de edad entre los 11 y 18 años del informante identificado y los hermanos adolescentes, y e) participación voluntaria de los informantes y sus familias.

\section{Participantes}

Fueron 21 participantes en total: tres mujeres, dos de ellas con diagnóstico de Leucemia Linfoblástica Aguda (Lla), la otra con diagnóstico de Leucemia Aguda Bifenotípica (LAB) y un hombre con diagnóstico 
de Leucemia Linfoblástica Aguda (LLA); sus edades oscilaban entre 13 y 16 años al momento del estudio. Participaron además cinco padres (tres mujeres y dos hombres), un abuelo y una abuela paterna, así como una tía paterna (tutores de uno de los informantes); también se incluyeron a cinco hermanas y cuatro hermanos (ver tabla 1).

\section{Instrumentos}

Para la recolección de datos se utilizó una técnica de tipo interrogativo mediante entrevistas semiestructuradas; se usó una guía que incluyó los siguientes ejes: a) composición familiar (datos sociodemográficos, números de miembros que conforman la familia, edad, nivel de escolaridad, ocupación, etc.), b) antecedentes familiares (tipo de enfermedades que ha habido en la familia), c) información acerca del diagnóstico (inicio de la enfermedad, pronóstico de curación, tratamiento y evolución), y d) funcionamiento familiar (roles, jerarquías de poder, límites y resolución de problemas por parte de la familia ante la aparición de la enfermedad). También se realizaron genogramas sólo para ilustrar la composición familiar y las relaciones de la familia, tanto previas como posteriores al diagnóstico de leucemia en el adolescente (McGoldrick \& Gerson, 1987).

\section{Procedimiento}

Dicha recolección de datos se realizó en cuatro fases: 1) se obtuvo autorización del HCGJIM para realizar el estudio, en cumplimiento del Capítulo II, Artículo 16 de La Ley General de Salud, que señala que en las investigaciones en seres humanos se protegerá la privacidad del individuo sujeto de investigación, identificándolo sólo cuando los resultados lo requieran y éste lo autorice. Por ello se protegió la identidad de los participantes, otorgando un seudónimo a cada familia y un número a cada informante identificado. La recolección se llevó a cabo del 20 de enero al $1^{\circ}$ de octubre del 2010;2) se contactó a las familias y se programaron citas para iniciar las entrevistas; 3 ) en entrevista familiar se obtuvieron datos generales acerca de composición familiar, información del diagnóstico, tratamiento, evolución y se hizo el trazado del genograma; también se elaboró un expediente por cada familia; 4) en entrevistas individuales, se profundizó en el funcionamiento fami- liar antes y después del diagnóstico de leucemia en el adolescente hasta saturar el tema y se corroboró la información obtenida en la entrevista familiar previa. Las entrevistas fueron audiograbadas y transcritas bajo previa autorización de los participantes.

El análisis de los datos obtenidos de las entrevistas fue de tipo temático (Krippendorff, 1990) y se hizo en cuatro pasos: 1) trazado de segmentos y elaboración de códigos abiertos, que se nombraron según aparecían al momento de la lectura flotante de renglón por renglón; 2) se diferenciaron los códigos sobre temas que aludían a los límites entre subsistemas, la jerarquía de poder, los roles y la resolución de problemas por parte de la familia ante la aparición de la enfermedad; 3) la construcción de familias de códigos se hizo mediante la separación de dos grandes temas: el funcionamiento familiar antes del diagnóstico (FFAD) y el funcionamiento familiar después del diagnóstico (FFDD). En el primero se identificaron los siguientes subsistemas: jerarquía de poder, límites, roles y resolución de problemas por parte de la familia ante la aparición de la enfermedad. El segundo tema incluyó: jerarquía de poder, límites, roles y resolución de problemas por parte de la familia ante la aparición de la enfermedad, y 4) la inferencia se realizó mediante interpretaciones apoyadas en la literatura y la construcción de las categorías sobre el funcionamiento familiar anterior y posterior al padecimiento. Esta serie de pasos fueron realizados con el apoyo del software Atlas-ti (Muhr, 1997) entre junio y octubre de 2010.

\section{RESULTADOS}

Los resultados se exponen en dos momentos: funcionamiento familiar antes del diagnóstico (FFAD), compuesto por las cuatro familias participantes, y después del diagnóstico de leucemia; cuya sección se compone sólo de tres familias debido al fallecimiento de la informante identificada con el número 3 y la declinación de la familia a seguir participando en la investigación. 
Tabla 1. Composición familiar de los participantes

\begin{tabular}{|c|c|c|c|c|c|}
\hline $\begin{array}{l}\text { Informante No. 1, con } \\
\text { diagnóstico de Leucemia } \\
\text { Linfoblástica Aguda en } \\
\text { noviembre de } 2009\end{array}$ & Edad & Sexo & Escolaridad & Ocupación & $\begin{array}{l}\text { Referencia de algún } \\
\text { familiar enfermo }\end{array}$ \\
\hline Hijo & 13 & $\mathrm{~F}$ & 6to. de primaria & Escolar & \multirow{7}{*}{$\begin{array}{l}\text { - Abuelo materno: } \\
\text { Tuberculosis } \\
\text { - Tío materno: Cáncer } \\
\text { pulmón } \\
\text { - Tía de la madre: } \\
\text { - Cáncer cervicouterino } \\
\text { - Tío paterno: Tumores } \\
\text { de cáncer no } \\
\text { especificados (finado) }\end{array}$} \\
\hline Madre & 49 & $\mathrm{~F}$ & Primaria & Hogar & \\
\hline Padre & 46 & $M$ & Secundaria & Preso* & \\
\hline Hermana & 29 & $\mathrm{~F}$ & Secundaria & Empleada & \\
\hline Hermana & 25 & $\mathrm{~F}$ & Secundaria & Empleada & \\
\hline Hermana & 23 & $\mathrm{~F}$ & Secundaria & Hogar & \\
\hline Hermana & 11 & $\mathrm{~F}$ & 5to. Primaria & Escolar & \\
\hline \multicolumn{6}{|l|}{ *Privado de su libertad desde 2007} \\
\hline $\begin{array}{l}\text { Informante No. 2, con } \\
\text { diagnóstico de Leucemia } \\
\text { Linfoblástica Aguda en enero } \\
\text { de } 2010\end{array}$ & Edad & Sexo & Escolaridad & Ocupación & $\begin{array}{l}\text { Referencia de algún } \\
\text { familiar enfermo }\end{array}$ \\
\hline Hija & 15 & $\mathrm{~F}$ & 2do. Secundaria & Estudiante & \multirow{4}{*}{$\begin{array}{l}\text { - Tía paterna: Leucemia } \\
\text { (finada) } \\
\text { - Abuela paterna: Cáncer } \\
\text { de estómago (finada) } \\
\text { - Abuelo paterno: } \\
\text { Tumor de cáncer no } \\
\text { especificado } \\
\text { - Bisabuela materna: } \\
\text { Diabetes }\end{array}$} \\
\hline Madre & 30 & $\mathrm{~F}$ & Primaria & Hogar & \\
\hline Padre & 40 & $M$ & 5to. Primaria & Campesino & \\
\hline Hermano & 13 & M & 2do. Secundaria & Estudiante & \\
\hline $\begin{array}{l}\text { Informante No. 3, con } \\
\text { diagnóstico de Leucemia Aguda } \\
\text { Bifenotípica en Febrero de } 2010\end{array}$ & Edad & Sexo & Escolaridad & Ocupación & $\begin{array}{l}\text { Referencia de algún } \\
\text { familiar enfermo }\end{array}$ \\
\hline Hijo & 15 & $\mathrm{~F}$ & 3ro. Secundaria & Estudiante & \multirow{6}{*}{$\begin{array}{l}\text { - Primo hermano del } \\
\text { padre: Cáncer no } \\
\text { especificado (finado) } \\
\text { - Tío paterno: Diabetes } \\
\text { - Bisabuela paterna: } \\
\text { Diabetes } \\
\text { - Por parte de la } \\
\text { familia del padre: } \\
\text { Enfermedades del } \\
\text { corazón, colesterol alto. } \\
\text { - Abuela materna: } \\
\text { Diabetes } \\
\text { - } 2 \text { tíos maternos: } \\
\text { Diabetes }\end{array}$} \\
\hline Madre & 39 & $\mathrm{~F}$ & Secundaria & Hogar & \\
\hline Padre & 44 & $M$ & Primaria & Campesino & \\
\hline Hermano & 19 & $M$ & Preparatoria & Campesino & \\
\hline Hermano & 17 & $M$ & Preparatoria & Campesino & \\
\hline Hermano & 11 & $M$ & 6to. Primaria & Escolar & \\
\hline
\end{tabular}




\begin{tabular}{|c|c|c|c|c|c|}
\hline $\begin{array}{l}\text { Informante No. 4, con } \\
\text { diagnóstico de Leucemia } \\
\text { Linfoblástica Aguda en Enero } \\
\text { de } 2010\end{array}$ & Edad & Sexo & Escolaridad & Ocupación & $\begin{array}{l}\text { Referencia de algún } \\
\text { familiar enfermo }\end{array}$ \\
\hline Hijo & 16 & M & $\begin{array}{c}\text { 3ero. } \\
\text { Secundaria }\end{array}$ & Estudiante & \multirow{8}{*}{$\begin{array}{l}\text { - Abuela Paterna: } \\
\text { Diabetes } \\
\text { - Tío abuelo: Diabetes } \\
\text { - Prima paterna: } \\
\text { Leucemia (finada) } \\
\text { - Abuelo paterno: Cáncer } \\
\text { de garganta } \\
\text { - Bisabuela paterna: } \\
\text { Alzheimer } \\
\text { - Bisabuelo paterno: } \\
\text { Leucemia (finado) }\end{array}$} \\
\hline Madre & 32 & $\mathrm{~F}$ & $\begin{array}{c}1 \text { ero. } \\
\text { Preparatoria }\end{array}$ & Hogar & \\
\hline Padre & 37 & M & Carrera Técnica & Empleado & \\
\hline Hermana & 15 & $\mathrm{~F}$ & $\begin{array}{c}2 \text { do. } \\
\text { Preparatoria }\end{array}$ & Estudiante & \\
\hline Hermano (2) & 11 & M & 6to. Primaria & Escolar & \\
\hline Abuelo paterno & 72 & M & Primaria & Jubilado & \\
\hline Abuela paterna & 68 & $\mathrm{~F}$ & 2do. Primaria & Hogar & \\
\hline Tía paterna & 41 & $\mathrm{~F}$ & Posgrado & Empleada & \\
\hline
\end{tabular}


Funcionamiento familiar antes del diagnóstico

\section{Jerarquía de poder}

En el FFAD se encontró que la familia López presenta jerarquía y límites claros entre los subsistemas; por ejemplo, cuando la mamá (de 30 años de edad) dice: Sí, la disciplina sigue, siempre ha sido igual como le digo [a su esposo], aquí hay un permiso, que quiere ir a tal lado pero pues, ¿cómo ves?, hay el acuerdo de los dos. En dos familias (Godínez y Pérez) se refirió que la jerarquía no es clara. En la familia Godínez el rol de autoridad lo comparten los abuelos paternos y la tía paterna.

Por otra parte, en la familia Pérez la jerarquía aparece poco clara cuando la madre (49 años) menciona que resulta habitual la participación de sus hijas en lo relacionado a la toma de decisión en actividades cotidianas relacionadas con el funcionamiento del hogar.

\section{Los límites: entre la rigidez y la flexibilidad}

En lo que se refiere a los límites, dos familias los presentan de forma difusa. Una de ellas es la familia Pérez, que describe la nula asignación de responsabilidades en el hogar. Al respecto, una de las hermanas (de 25 años de edad) comentó: "Pues no hay límites porque mi mamá nunca ha sido de las personas estrictas que diga: haces esto y haces esto porque lo haces. No, nunca fue asi mi mamá". En la familia Godínez los límites aparecieron difusos; por un lado, con las mujeres de la familia resultaron rígidos y con los hombres eran más flexibles, o no existían; la hermana (de 16 años de edad) refiere que su hermano tenía permiso para llegar más tarde que ella por el hecho de ser mujer; este tipo de pautas de interacción también se presentaban entre los abuelos paternos y sus hijos, lo cual habla de características propias de las familias rígidas, donde generalmente los padres no están preparados para cambiar las normas y reglas establecidas hacia los hijos pequeños y los problemas aparecen, por lo común, cuando los padres se oponen a los cambios necesarios para que los hijos desarrollen habilidades en la toma de decisiones $\mathrm{y}$ de autosuficiencia.

\section{Los roles: el desarrollo de los oficios}

En cuanto a los roles familiares, se observó que en tres de las familias participantes existe la distribución de tres funciones acordes con el rol de cada miembro de la familia: 1) los papás trabajaban; 2) las mamás se hacen cargo de labores en el hogar; y 3) los hijos estudian y/o trabajan; cabe señalar que dos de estas familias proceden de dos poblados muy pequeños del estado de Michoacán. En la familia Pérez, la madre asumió la función del sustento económico, apoyada por sus hijas mayores, y el abuelo materno de la informante identificada, a partir de que el padre ingresó al Centro de Readaptación Social.

Resolución de problemas por parte de la familia ante la aparición de la enfermedad

La capacidad de resolución de problemas se vio reflejada en dos familias. Dos acciones aparecieron como las recurrentes: a) la búsqueda de atención médica para sus hijos. Los miembros de la familia González describieron que el primer contacto realizado fue con un médico general que los envió con otro profesional; posteriormente, establecieron una cita con otro médico, obteniendo nulos resultados, ya que no propició el seguimiento del caso; por último, los padres solicitaron una cita con un especialista que los envió al Hospital Civil de Guadalajara para realizar estudios más especializados. Y b) la búsqueda de solvencia económica mediante trabajo remunerado. En el caso de la familia Pérez, cuando el padre de la informante número 1 ingresó al Centro de Readaptación Social, la madre decidió cambiar de residencia junto con sus hijas a casa de su padre; su decisión obedeció a la necesidad de cubrir los gastos de alimentación y de vestido de ella y sus hijas; la madre se dedicaba a cuidar a sus nietos y ayudaba a sus hijas mayores mientras ellas trabajaban en la estética.

\section{Funcionamiento familiar después del diagnóstico}

\section{Jerarquía de poder}

Las jerarquías entre subsistemas resultaron iguales que las previas al diagnóstico en dos de las familias. Una de ellas fue la familia López: la madre (de 30 años de edad) describió cómo los permisos siempre los concedían entre ella y su esposo, tomando ella el rol de negociadora entre él y sus hijos cuando había renuencia por parte del primero para otorgar algún permiso en específico. 
Figura 1. Genograma de la "familia Pérez" que ilustra las relaciones familiares antes del diagnóstico de leucemia

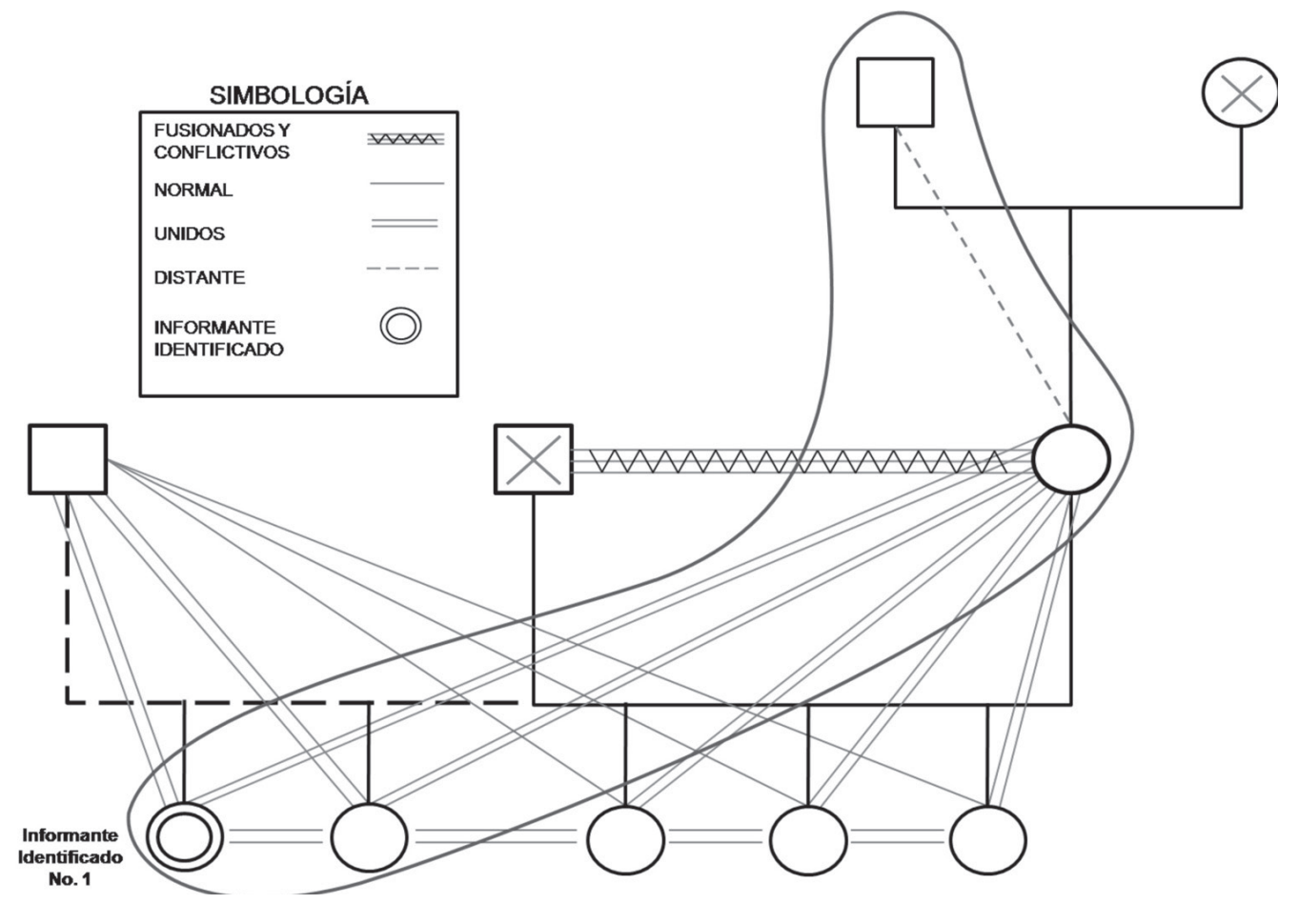

Figura 2. Genograma de la familia Godínez que ilustra las relaciones familiares antes del diagnóstico de leucemia

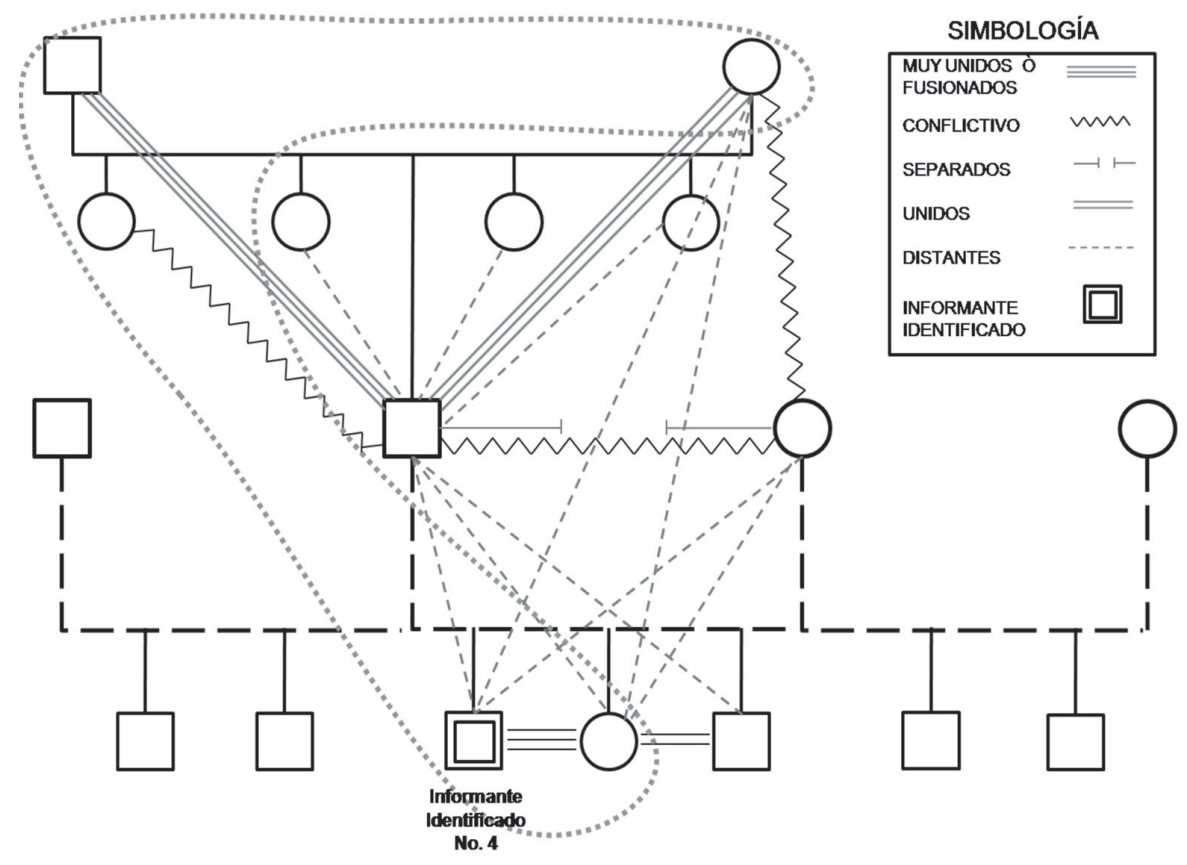


Figura 3. Genograma de la familia López que ilustra las relaciones familiares antes del diagnóstico de leucemia

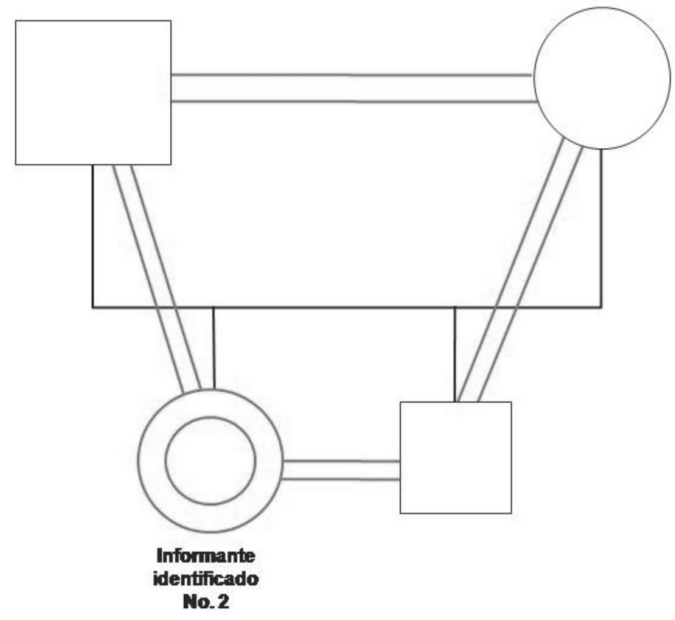

SImBOLOGiA

undos

IMFopanwIE IDENIIPCADO

Figura 4. Genograma de la familia Godínez que ilustra las relaciones familiares después del diagnóstico de leucemia

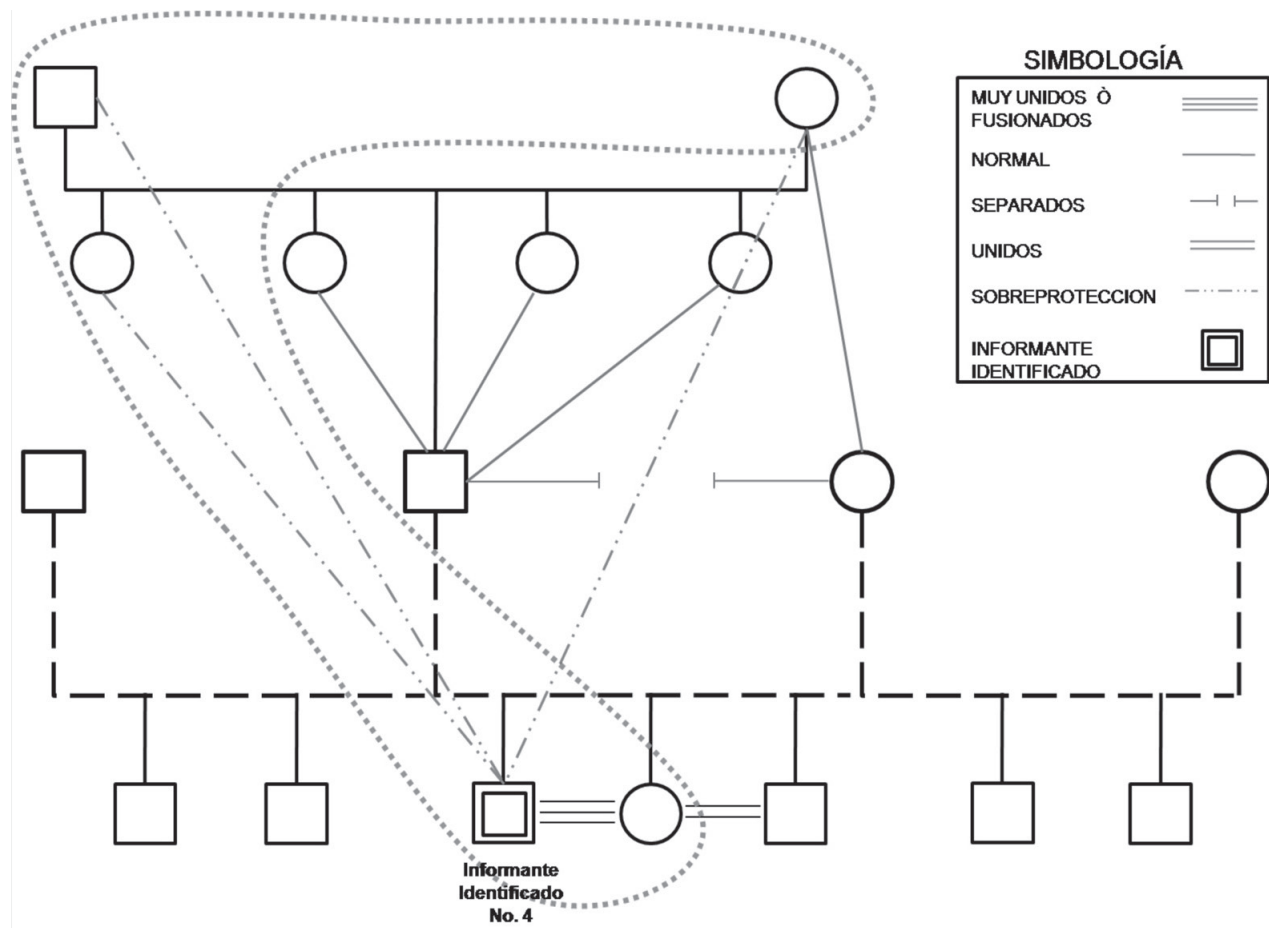


Tampoco hubo cambios en la familia Pérez: la madre refirió que cuando sus hijas mayores eran pequeñas ella tenía que trabajar, debido a esto no ejercía plena autoridad en las actividades cotidianas del hogar y en el cuidado de las niñas, lo que facilitó que fueran autosuficientes desde muy pequeñas.

Para la familia Godínez, la jerarquía apareció difusa y/o rígida; se observó que la aparición de la enfermedad produjo cambios, ya que el informante número 4 describió que su familia le prestaba mayor atención y se preocupaba más por él desde que se enfermó; lo mismo describe la hermana (de 15 años de edad): "Pues, como que ya la atención, este, como que la prioridad es él”. También se percibió mayor flexibilidad con respecto a la posición de autoridad de los abuelos hacia el informante; la abuela (de 68 años de edad) relata que siempre le llamaban la atención por molestar a los nietos más pequeños pero él nunca entendió.

\section{Límites}

Se encontraron cambios en la familia Godínez, ya que los límites aparecieron más flexibles a partir del diagnóstico de la enfermedad. La asociación entre los límites difusos y/o rígidos y actitudes de sobreprotección hacia el hijo enfermo, aparecieron frecuentemente en esta familia. Estas pautas se presentaron sobre todo en la abuela y tía paterna, tal como lo refiere la hermana (de 15 años de edad): "A mí me gustaría que mis tías y mi abuela no lo sobreprotegieran tanto, porque yo siento que después ya no va a querer hacer nada, ni estudiar ni nada y a mí me da miedo porque, después digo: ¿qué va a ser de él?".

En las familias con límites claros se encontró una relación directa y positiva con el autocuidado, ya que al permitir que el hijo enfermo participara activamente en el tratamiento, éste se responsabilizaba de horarios, medicamentos, citas, etc. Una de las familias donde se

\section{Figura 5. Genograma de la familia Pérez que ilustra las relaciones familiares después del diagnóstico}

de leucemia

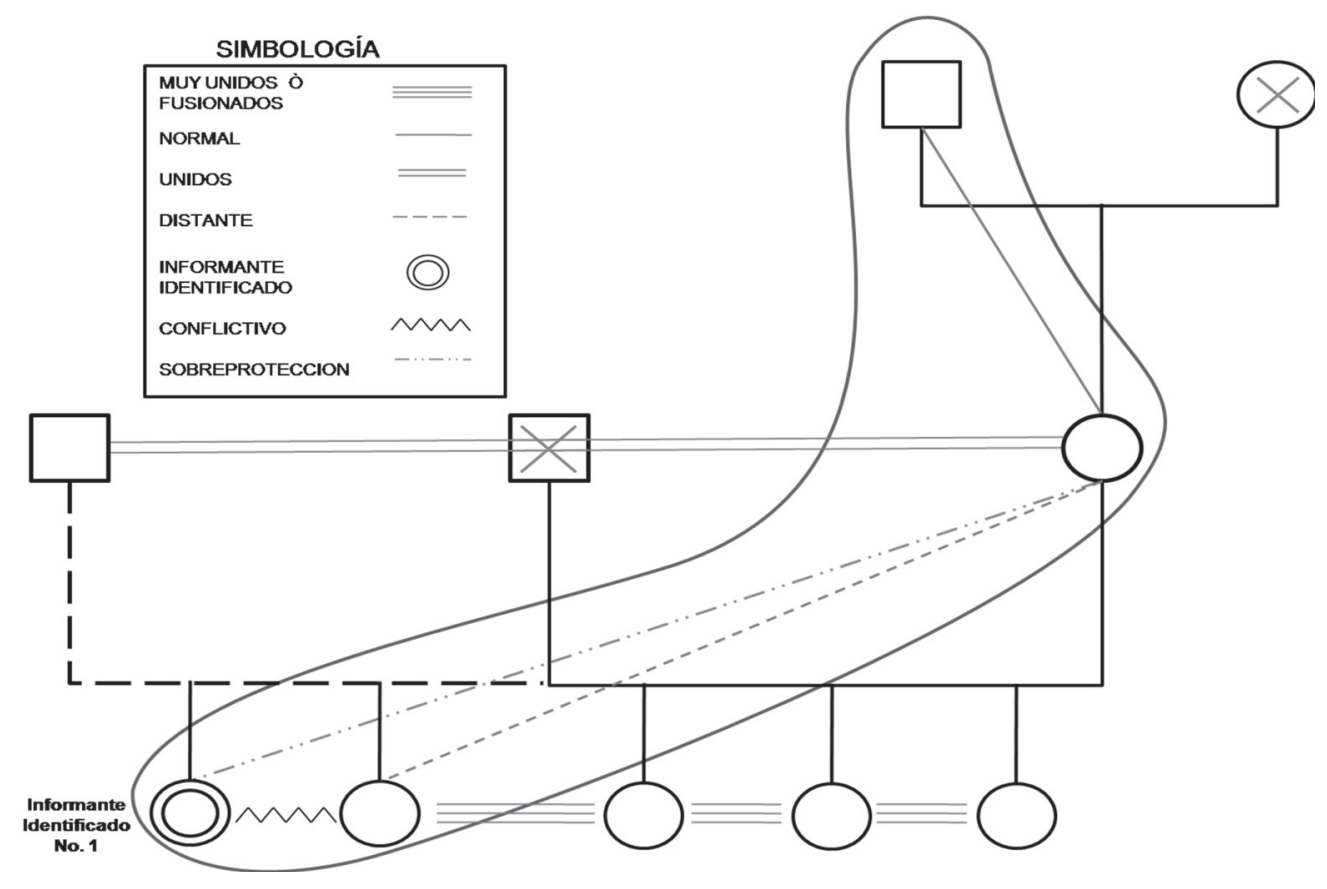


percibió este tipo de pautas fue la familia Pérez, lo cual produjo en la informante número 1 una participación activa y directa en la adherencia a su tratamiento.

\section{Roles}

En lo referente a los roles, observamos dos tipos de cambios en las familias participantes: 1) el abandono de sus actividades habituales, como inasistencia o renuncia al trabajo, la realización de las labores del hogar o la permanencia en las actividades escolares. Al respecto, la familia López relató que todos los miembros del sistema se trasladaban cuando asistían a tratamiento médico, aunque algunos permanecían en el hospital $y$ otros en casa de familiares para retornar juntos al término del mismo; 2) la centralización de las actividades sociales y recreativas en el hijo enfermo, quien se convirtió en el centro de atención de los padres, éstos deciden y priorizan las actividades familiares en función del estado de salud y anímico del mismo; por ejemplo, el tipo de comida que él prefiera o las salidas y reuniones familiares donde y cuando él guste llevarlas a cabo.

Resolución de problemas por parte de la familia ante la aparición de la enfermedad

En la familia López el padre (40 años) refirió que en cuanto a la economía tenía más problemas antes de la enfermedad de su hija; sin embargo, su discurso es reiterativo acerca de las dificultades que ahora atraviesa para conseguir dinero cuando la informante número 2 tiene que acudir al hospital a recibir tratamiento.

\section{DISCUSIÓN}

Según la descripción de las pautas familiares en el sistema familiar, éstas se clasifican en antes y después del diagnóstico de leucemia en el adolescente, en función de: jerarquías, límites, roles y resolución de problemas por parte de la familia ante la aparición de la enfermedad; en tres de las familias participantes prevaleció un funcionamiento familiar adecuado con organización jerárquica, roles y límites claros, así como una estructura flexible. En el caso de una familia, encontramos evidencia clara de un funcionamiento familiar de tipo disfuncional en cuanto a jerarquías indefinidas, roles y límites difusos.

En relación con la jerarquía, las familias funcionales mostraron claridad en el ejercicio de la autoridad, ya que las decisiones dentro del hogar estuvieron a cargo de los padres y/o tutores aún después del diagnóstico. Una posible explicación radica en que la diferenciación entre generaciones en estas familias es clara; según Minuchin (1985) y Navarro (2004), en la cultura occidental se espera que los padres tengan más poder

Figura 6. Genograma de la familia López que ilustra las relaciones familiares después del diagnóstico de leucemia
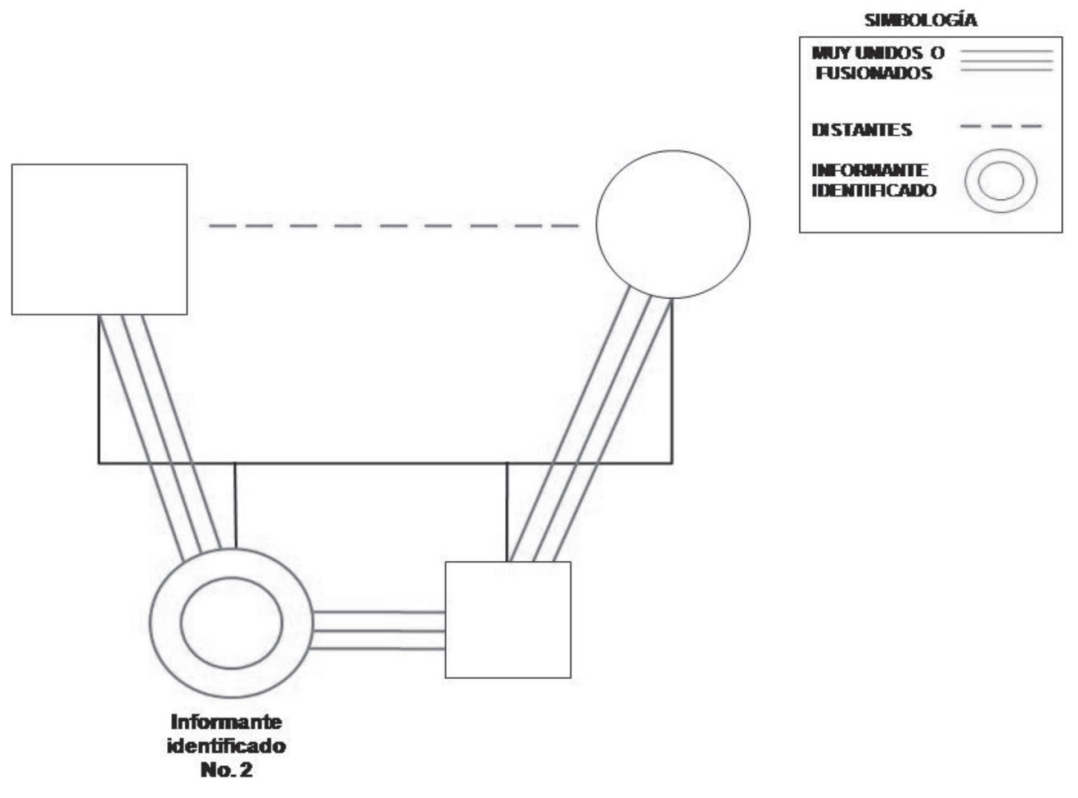
que los hijos. Por ende, una jerarquía clara como se identificó antes y después del diagnóstico en dos de las familias participantes, se caracteriza por la existencia de normas establecidas por los padres para responsabilizarse de sus hijos y que éstos cooperen con ellos (Die-Trill, 1987). En algunas de las familias de este estudio se encontró una jerarquía invertida después del diagnóstico de la enfermedad, lo que se reflejó en el hecho de que los padres fueron más flexibles con el hijo enfermo y más exigentes con los otros hijos. En el caso de una de las familias la jerarquía se mantuvo difusa, ya que la autoridad fue depositada en los miembros de la familia extensa. Lo anterior habla del estilo de respuesta nombrada tendencia centrífuga, que se manifiesta por conductas evitativas de los miembros de la familia con respecto a la situación de enfermedad, donde uno de los miembros se hace cargo del cuidado del enfermo, controlándole en exceso, coartando su autonomía y quitándole la responsabilidad de hacerse cargo de su evolución, mientras los demás miembros se despreocupan de esa situación (Kornblit, 1984); según la misma autora, en la mayoría de las familias la enfermedad ejerció una influencia centrípeta, ya que desarrollaron o fortalecieron lazos de unión y cooperación.

En lo referente a límites y reglas existentes en el FFDD de leucemia en el adolescente, se encontró que la enfermedad exige un reordenamiento a cada uno de los miembros del sistema. Algunas familias del estudio poseen límites claros en el subsistema conyugal, lo cual indica la presencia de un adecuado funcionamiento ante las situaciones críticas (Kornblit, 1984; Minuchin, 1985). En este mismo subsistema, algunas parejas poco funcionales presentaron cambios permanentes, produciendo estabilidad en la pareja.

En las familias que comparten el rol parental entre la madre y otros miembros de la familia extensa, se observaron límites difusos con tendencia a la flexibilidad. Estos hallazgos indican que dichas familias presentan límites polarizados con una tendencia a la difusión. De acuerdo con Minuchin (1985), lo anterior no favorece la funcionalidad para la crianza de los hijos. En contraste, uno de los estudios revisados, menciona que la presencia de límites difusos y rígidos prevalece en familias con un subsistema conyugal poco funcional o no existente; así como en familias donde la madre asume los cuidados del hijo enfermo y cuyo padre sólo apoya económicamente (Meléndez, 2005).

En este estudio algunos padres que ejercen límites difusos experimentan impotencia ante la amenaza de la enfermedad de su hijo, teniendo que delegar la función parental de protección al equipo de salud (médicos, psicólogos y enfermeras). Estos cambios pueden generar sentimientos ambivalentes por parte de los hermanos del paciente, ya que por un lado, sienten enojo y celos porque se le da un trato privilegiado y porque toda la atención de los padres está dirigida al informante identificado, y por otro, sentimientos de soledad y culpa por albergar este tipo de emociones hacia su hermano enfermo; al respecto, Méndez (2007) y Velasco y Sinibaldi (2001) refieren que dichos cambios pueden ser más graves si los hermanos no cuentan con información fiable que explique la actitud de los padres.

En este sentido, Velasco y Sinibaldi (2001) mencionan que resulta común la presencia de preocupación, ansiedad y sentimientos de culpa en los hermanos acerca de las causas y el pronóstico de la enfermedad, así como el temor de que ellos también la padezcan. Otros estudios informan que un alto porcentaje de los hermanos de niños leucémicos presentan síntomas psicológicos, trastornos somatoides, o ambos, como fobia escolar, dolores de cabeza, depresión y ansiedad por separación (Muñoz \& Paredes, 2006; Ramírez, 2008). Razavi hace énfasis en la importancia de tener en cuenta mientras más jóvenes sean, mayor será el grado de afectación de los hijos no enfermos a la hora de entender las respuestas del núcleo familiar, porque su entendimiento de la situación es menor; estos problemas muchas veces no son detectados por los padres, quienes se enfrascan en el hijo enfermo y sus propias dificultades (Barreto et al., 2000).

Una posible explicación acerca de la presencia de límites difusos radica en el ejercicio de la sobreprotección hacia el adolescente con leucemia, ya que se observan diferencias en el ejercicio de la crianza y la autoridad entre el adolescente enfermo y los otros hijos, presentándose mayores dificultades hacia los primeros. Navarro y Beyebach (1995) refieren que la evaluación de la enfermedad crónica facilita cambios estructurales en los patrones de interacción familiar; la sobreprotección es uno de éstos, ya que tiene consecuencias que dificultan la situación. 
En lo que respecta a los roles familiares, los cambios encontrados después del diagnóstico obedecieron a las necesidades específicas de cada familia; en todas aparece el rol de "cuidador", que recae tanto en el subsistema parental como en el fraterno. Al respecto, según Micnuchin (1985), se puede afirmar la existencia de complementariedad como una de las principales cualidades del sistema familiar, que permite a cada miembro de dichos subsistemas, sobre todo del parental, ceder sin sentir que pierde su estatus. Para Velasco y Sinibaldi (2001) este intercambio de roles manifiesta una adecuada fluidez y capacidad para el intercambio de funciones, así como el deseo de compartir algunas de ellas entre los miembros.

Nuestros hallazgos coinciden con los de Ramírez (2008), Meléndez (2005) y Muñoz y Paredes (2006), en relación con que se observó que los padres se dan cuenta de la necesidad de reorganización y planificación de actividades a nivel individual, conyugal, parental, fraternal y familiar después del diagnóstico de la enfermedad, lo que implica poner en juego una serie de recursos internos del sistema y realizar cambios.

En lo que respecta a la resolución de problemas por parte de las familias ante la aparición de la enfermedad, se privilegió la búsqueda de ayuda profesional por encima de creencias distorsionadas sobre la etiología de la misma. Aunque algunos movimientos familiares no resultaron exitosos en la búsqueda de ayuda, ya que prevalecían interacciones distantes y jerarquías difusas, es notable que las progenitoras y algunos padres emprendieran el contacto con los servicios de salud adecuados para el tratamiento del cáncer, además de encontrar disposición por parte de estos últimos.

Pese a que estas familias se observan funcionales, la aparición de una enfermedad provoca cambios radicales en el funcionamiento emocional del día a día de los miembros y se da una ruptura de la rutina normal; se incrementan los niveles de estrés, tal como se identificó en algunas familias de este estudio. Es pues predecible que si existían problemas familiares antes de la aparición de la enfermedad, estos se agudicen tras el diagnóstico, ya que también el nivel de estrés que afecta a la familia puede favorecer la aparición de nuevos problemas (Minuchin, 1985).

Las fortalezas y aportes de esta investigación radican en el análisis del funcionamiento de las familias participantes a través de la estructura familiar en cuanto a sus pautas e identificación de los cambios que se producen en el sistema familiar ante el evento de la enfermedad, así como la característica homeostática del sistema para reorganizarse y adoptar el equilibrio como un nuevo aprendizaje a partir de la enfermedad. Con base en los hallazgos de esta investigación, podemos considerar que las familias nucleares, reconstituidas y extensas de este estudio podrían contextualizar la modernidad mexicana, la cual refleja que los tipos de familias y sus costumbres atraviesan por la vivencia de una enfermedad crónica fundamentada en la teoría familiar sistémica, sobresaliendo el proceso de reorganización familiar en su interior.

Este trabajo contribuye al estudio del cáncer en la familia desde una perspectiva sistémica de primer orden, reconociendo la sintomatología de la enfermedad y el funcionamiento de la familia para hacer frente al padecimiento, además de que pone en evidencia las posibles pautas de tratamiento en la terapia familiar para ser integradas en el trabajo multidisciplinario de los servicios oncológicos. Aunque resulta ampliamente documentado que el enfoque estructural ha logrado avances en la transformación de pautas disfuncionales en la familia, este estudio coadyuva a la integración de diferentes miradas en el tratamiento psicoterapéutico de la familia, principalmente incluir el punto de vista de los miembros que acompañan a quien padece $y$, a quien se percibe como enfermo de cáncer.

Otro aporte de este trabajo es la profundidad alcanzada en las familias con adolescentes enfermos de leucemia participantes, lo cual resulta relevante para comprender la realidad de las necesidades de estas familias mexicanas; lo anterior es importante debido a que en la fase de revisión bibliográfica se pudo constatar que son escasos los estudios nacionales de familias con niños o adolescentes que padecen leucemia o cualquier tipo de cáncer bajo un enfoque sistémico.

Asimismo, se advierten algunas limitaciones en esta investigación: el investigador puede encontrar poca disponibilidad de los adolescentes que padecen algún tipo de cáncer; en el caso específico de este estudio, leucemia, por la exacerbación de sus síntomas o por característica propia de la adolescencia; también se encontrará con el débil involucramiento de algunos miembros de las familias, que si bien no cohabitaban 
directamente con el enfermo, podrían aportar datos sobre algunas pautas de relación poco estudiadas.

Otra limitación alude a la poca profundización en otras dimensiones involucradas en el funcionamiento familiar, como son los tipos de comunicación y sus axiomas que ilustran el intercambio verbal y no verbal, adaptabilidad, cohesión y los recursos de afrontamiento, incluido el apoyo social; tampoco se analizó el apoyo emocional que brinda cada uno de los miembros de la familia y su impacto en el afrontamiento general de la enfermedad del cáncer. Lo anterior constituye una futura implicación de este estudio debido a lo extenso de los resultados y su análisis.

En el inicio de la enfermedad, el diagnóstico y la cronicidad del padecimiento, las familias con adoles- centes que padecen leucemia presentan alteraciones en su funcionamiento familiar. Antes de la enfermedad se identificaron límites y jerarquías claras, roles funcionales y fronteras flexibles en las familias. Después, el diagnóstico y el curso de la enfermedad propiciaron la presencia de jerarquías y límites difusos, así como roles débilmente claros, provocando una desorganización del sistema familiar cuya duración fluctúa de uno a dos meses aproximadamente, asimsimo se integraron nuevas pautas en forma de resolución de problemas por parte de la familia ante la aparición de la enfermedad. Por último, se presentó una fase de adaptación y reorganización del sistema mediante el empleo de pautas funcionales, así como de los miembros de la familia, y el apoyo de las instituciones de salud.

\section{REFERENCIAS}

Aguilar, B. A. (2007). Factores psicológicos y sociales en las neoplasias malignas pediátricas. Revista Cubana de pediatría, 79(1). Recuperado el 20 de noviembre del 2008, de: http://scieloprueba.sld. $\mathrm{cu} /$ scielo.php? script $=$ sci_arttext\&pid=SO034 $75312007000100001 \&$ Ing $=\&$ nrm $=$ iso\&ting $=$

Atri, R. (2006). Cuestionario de Evaluación del Funcionamiento familiar (EFF). En M. Velasco \& M. Luna (Eds.). Instrumentos de evaluación en terapia familiar $y$ de pareja (1-27). México: Pax.

Barreto, M., Molero, M. y Pérez, M. (2000). Evaluación e intervención psicológica en familias de enfermos oncológicos. En F. Gil (Ed.). Manual de psico-oncología (135-182). Madrid: Nova Sidonia Oncología.

Caqueo A. \& Lemos, S. (2008). Calidad de vida y funcionamiento familiar de pacientes con esquizofrenia en una comunidad latinoamericana. Psicothema, 20, 4, 577-582. Recuperado el 17 de julio del 2009 de: http://redalyc.uaemex.mx/src/inicio/ArtPdfRed. jsp?iCve $=72720411$

Ccoicca, T. (2010). Bullying y funcionalidad familiar en una institución educativa del Distrito de Comas. (Tesis de licenciatura no publicada). Universidad Nacional Federico Villarreal, Facultad de Psicología. Lima, Perú. Recuperado el 18 de Mayo del 2009 de: http://www.observatorioperu.com/imagenes/teofiloccoiccabullyingyfuncionalidadfamiliar.pdf

Cuevas, U., Villasis, K., \& Fajardo, G. (2003). Epidemiología del cáncer en adolescentes. Revista de Salud Pública de México, 45(1), 115-123.

Die-Trill, M. (1987). Niños y adolescentes con cáncer: aspectos psicológicos. Revista Humanidades Médicas Psicología y Medicina, Madrid. 33, 35-41.

Fajardo, G., Mejía, A., Hernández, C., Mendoza, S., Garduño, E. \& Martínez, G. (1999). Epidemiología descriptiva de las neoplasias malignas en niños. Revista Panamericana de Salud Pública, 6(2), 75-88.

Fernández, A. \& Musito, O. (2007). Teoría de sistemas: Adolescencia y familia. Universidad de León: Secretariado de publicaciones.

Gasulla, J. (2009). El modelo biopsicosocial de enfermedad. ¿Por qué 3, y no 2 o 1 ? Hospital General I'Hospitalet de Llobregat. Barcelona. Interpsiquis. Recuperado el 4 de febrero del 2010, de: http://www. psiquiatria.com/articulos/psiq_general_y_otras_ areas/psicosomatica/38984/

Herrera, P. (1997). La familia funcional y disfuncional, un indicador de salud. Revista Cubana Medicina General Integral, 13(6), 591-595. Recuperado el 30 de abril del 2008, de: http://bvs.sld.cu/revistas/mgi/ vol13_6_97/mgi13697.htm

Kornblit, A. (1984). Somática familiar, enfermedad orgánica y familia. Barcelona: Gedisa. 
Krippendorff, K. (1990). Metodología del análisis de contenido. Teoría y práctica. Madrid: Paidós Ibérica.

López, P., Sauceda, G. \& y Cuevas, U. (2006). Funcionamiento familiar y su relación con bajo rendimiento escolar, la adaptación psicosocial y psicopatología en adolescentes mayores. Revista Psiquis (México), 15,1, 13-23. Recuperado el 1 de diciembre del 2008, de: http://www.imbiomed.com.mx/1/1/articulos.php? method $=$ showDetail\&id_articulo $=47563 \& i d$ seccion $=3011$ \&id_ejemplar $=4822 \&$ \&id_revista $=180$

McGoldrick, M. y Gerson, R. (1987). Genogramas en la evaluación familiar. Barcelona: Gedisa.

Méndez, V. (2007). Aspectos psicológicos del niño con cáncer. En R. Rivera (Ed.). El niño con cáncer. Los padecimientos más comunes para el médico no especialista (275-289). México: Editores de textos mexicanos.

Meléndez, J. (2005). Estudio descriptivo y analítico relacional, acerca de la estructura familiar y creencias de familias con un hijo que porte una discapacidad física producida por un mielomeningocele. (Tesis de licenciatura no publicada). Universidad de Chile, Facultad de Ciencias Sociales, Carrera de Psicología: Santiago, Chile. Recuperado el 13 de enero del 2010 de: http:// www.cybertesis.cl/tesis/uchile/2005/melendez_j/ sources/melendez_j.pdf

Minuchin, S. (1985). Familias y terapia familiar. Buenos Aires: Gedisa.

Minuchin, S. \& Fishman, H. (1984). Técnicas de terapia familiar. Argentina: Paidós.

Muhr, T. (1997). Scientific Software Development's ATLAS-ti. Visual cualitative data. Version for windows 95 and windows NT. Berlín: Scientific Software Development.

Muñoz, E. \& Paredes, D. (2006). Estudio exploratorio sobre la percepción de los padres respecto al funcionamiento familiar, en los primeros meses, posterior a un diagnóstico de cáncer de Leucemia Linfoblástica Aguda (lla) en hijos, cuyas edades fluctúan entre los 2 $y$ los 9 años, de ambos sexos; pertenecientes a la unidad de Hemato-Oncología del Hospital Dr. Gustavo Fricke, de Viña del Mar. (Tesis de Licenciatura en Psicología). Recuperado el 10 de febrero del 2010, de: http://users.udelmar.cl/loyola/WEBs/psicologia/ data/upimages/familia_cancer.pdf
Navarro, J. (2004). Enfermedad y familia. Barcelona: Paidós Ibérica.

Navarro, J. \& Beyebach, M. (1995). Avances en terapia familiar sistémica. Barcelona: Paidós Ibérica.

Ortiz, M. (1999). La salud familiar. Revista Cubana Med Gen Integr 15(4), 439-445. Recuperado el 25 de abril del 2010, de: http://bvs.sld.cu/revistas/mgi/ vol15_4_99/mgi17499.pdf

Ortiz, M. \& Louro, I. (1996). Proyectos de intervención en salud familiar. Una propuesta metodológica. (Tesis de Maestría en Salud Pública). Recuperado el 7 de junio del 2010, de: www.sld.cu/galerias/doc/sitios/pdguanabo/bcfamiliar.doc

Pérez, C. \& Rodríguez, H. (2005). Repercusión familiar de un diagnóstico oncológico. Recuperado el 2 de diciembre del 2008, de: http://psicolatina.org/Tres/ repercusion.html

Ramírez, C. (2008). Funcionalidad familiar y enfermedad crónica infantil. Recuperado el 17 de julio del 2009, de: http://www.nuevoamanecer.edu.mx/imgs/pdf/ Funcionalidadfamiliar.pdf

Umbarger, C. (1983). Terapia familiar estructural. Argentina: Amorrortu.

Velasco, M. \& Sinibaldi, J. (2001). Manejo del enfermo crónico y su familia (sistemas, historias y creencias). México: Manual Moderno.

\section{Agradecimientos}

A los adolescentes con diagnóstico de leucemia de este estudio y sus familias. A la Q. F. B. Ma. Guadalupe Flores G., coordinadora de Oncología, División Farmacéutica de Laboratorios Pisa, por su interés en el área de psico-oncología, lo cual favoreció la obtención de resultados mediante su apoyo institucional.

Fecha de recepción: Enero 2012 Fecha de publicación: Junio 2012 